\title{
A childhood case of symptomatic essential and psychogenic palatal tremor
}

This article was published in the following Dove Press journal:

Neuropsychiatric Disease and Treatment

26 April 20II

Number of times this article has been viewed

\author{
Francesco Margari' \\ Giustina Giannella ${ }^{2}$ \\ Paola Alessandra Lecce ${ }^{2}$ \\ Piero Fanizzi ${ }^{3}$ \\ Maddalena Toto ${ }^{2}$ \\ Lucia Margari \\ 'Psychiatry Unit; ${ }^{2}$ Child \\ Neuropsychiatry Unit, Department \\ of Neurologic and Psychiatry \\ Sciences; ${ }^{3}$ Otorhinolaryngology Unit, \\ Department of Ophthalmology and \\ Otorhinolaryngology, "Aldo Moro" \\ University of Bari, Bari, Italy
}

Correspondence: Francesco Margari Azienda Ospedaliero-Universitaria Policlinico, Piazza Giulio Cesare II, 70124 Bari, Italy

Tel +39080 5478576

Fax +39080 5478549

Email margari.f@psichiat.uniba.it

\begin{abstract}
Palatal tremor is a rare movement disorder characterized by rhythmic contractions of the soft palate. It is most often symptomatic, secondary to brainstem or cerebellar disease and, in rarer cases, is categorized as essential in the absence of documented brain lesions. There have also been reports in the literature of cases of palatal tremor described as psychogenic because they were associated with psychological or psychiatric disorders. We describe the case of a 12-year-old boy with palatal tremor presenting clinical features of symptomatic essential and psychogenic palatal tremor, thus suggesting a neuropsychopathological continuum between the different forms of disease.
\end{abstract}

Keywords: palatal tremor, psychogenic movements, child movement disorders

\section{Introduction}

Palatal tremor is a rare movement disorder characterized by rhythmic contraction of the soft palate. Palatal tremor is most often symptomatic, secondary to brainstem or cerebellar disease and, in rarer cases, is categorized as essential in the absence of documented brain lesions. ${ }^{1}$ Some authors have documented hypertrophic degeneration of the inferior olives in secondary palatal tremor, but its precise role in causing palatal tremor has not yet been clarified and this finding is currently controversial. $^{2}$

Essential palatal tremor generally affects children of both genders, whereas secondary palatal tremor is most commonly observed in adult males. Essential palatal tremor is bilateral and usually disappears during sleep, whereas secondary palatal tremor is more frequently unilateral and persists even during sleep. Patients with essential palatal tremor usually have an ear click, which is absent in the symptomatic form.

Although essential palatal tremor has a benign course and usually disappears spontaneously, it is very annoying for the patient, so several treatments have been administered, including anticonvulsants and anxiolytics, with varying therapeutic effects. At present no specific treatment has been established, although successful treatment by botulinum has recently been reported. ${ }^{3}$

There have also been reports in the literature of cases of palatal tremor which have been described as psychogenic because they were associated with psychological or psychiatric disorders. In this form, known as psychogenic palatal tremor, the abnormal movement is intermittent, increases during attention, decreases during distraction, and is under voluntary control. ${ }^{4}$ We describe the case of a 12 -year-old boy with palatal tremor presenting clinical features of symptomatic essential and psychogenic 
palatal tremor, thus suggesting a neuropsychopathological continuum between the different forms of palatal tremor.

\section{Case report}

The case was a 12-year-old boy, the only child of unrelated parents. There was a positive family history of psychiatric disorders, in that the father has a personality with borderline and narcissistic traits, poor impulse control, and multiple motor tics, and the mother had a major, recurrent depressive disorder, and histrionic and paranoid personality traits. A maternal aunt suffered from a severe bipolar disorder. Moreover, the emotional climate in this family was characterized by a high level of "expressed emotion" with over involvement and criticism, and with a very conflictual relationship between the parents. ${ }^{5}$ The patient was born by elective cesarean delivery at the eighth month of gestation after a pregnancy characterized by gestational diabetes treated with insulin. He showed mild motor developmental delay and inadequate performance at primary school, and difficulty in relating to peers. He came to our attention about one year earlier due to the emergence of behavioral disturbances characterized by oppositional defiant behavior and aggression, episodes of psychomotor agitation with dysphoria, and aggression towards others. About two years before these events, when the patient was 10 years old, his parents had started to hear a clicking sound coming from the boy, but no research was carried out until our observations. No drug therapy had been taken.

We performed clinical and instrumental assessments, including physical, neurological, and otolaryngological examinations, laboratory tests, audiological and neuroimaging studies, and a psychodiagnostic assessment, including the IQ rating scale (Wechsler Intelligence Scale for Children III) and interviews administered to the patient and his parents using the Child Behavior Checklist and Schedule for Affective Disorders and Schizophrenia for School-Age Children - Present and Lifetime Version.

The neurological examination showed only hypotonia of the facial muscles causing drooling, but no other clinical signs, except for the presence of rhythmical bilateral palatal movements, mainly on the right side, associated with a clicking sound that could easily be heard when listening close to the child's ear. No ataxia or nystagmus was present. The clicking was continuous and audible by his parents even during sleep. The patient seemed to be very annoyed and concerned about this ear click, which was present with the mouth both open and closed. Nevertheless, it was under voluntary control, and the patient was able to voluntarily induce it or stop it for a few minutes. Moreover, the ear click was less audible or had a changed frequency during the performance of complex motor and cognitive tasks.

Otolaryngological examination with fibroscopy detected a bilateral palatal tremor at a frequency of 80 cycles per minute. Laboratory tests, including a complete blood count, antinuclear antibody studies, thyroid tests, plasma copper, ceruloplasmin, plasma amino acids, serum lactate and pyruvate, ammonium, and transaminases, were normal. Audiometry and electroencephalography were also normal, as were brain magnetic resonance imaging, angiographic magnetic resonance imaging, and proton magnetic resonance spectroscopy, focusing particularly on study of the brainstem.

During spontaneous observation, the patient showed a reduced adaptive and relational capacity, with his relationships being characterized by oppositional behavior that sometimes culminated in episodes of aggression and psychomotor agitation. His moods and affectivity were extremely unstable, featuring sudden swings and a poor control of emotions and impulses, in particular at moments of frustration and deferral of pleasure. Psychosomatic and anxiety symptoms were also detected. At the WISC-III test, he obtained an IQ of 65 (verbal IQ $=81$, performance IQ $=56$ ), expressing mild mental retardation.

During the previous year, several therapies had been attempted, including anticonvulsants and antipsychotic drugs, with variable results. Topiramate and valproic acid treatment showed no effect on either the behavioral disturbances or the palatal tremor, and carbamazepine therapy showed a transient effect that disappeared for about two months but then recurred. Due to an increased frequency of episodes of agitation, chlorpromazine therapy was started, then carbolithium was added, resulting in an improvement of the behavioral disturbances. Treatment with piracetam, the effectiveness of which in essential palatal tremor was recently reported by Campistol-Plana et al, ${ }^{3}$ was ineffective. It was not possible to administer treatment with botulinum toxin due to the patient's limited cooperation. All drug treatments were associated with psychotherapy. After six months of treatment with carbolithium, chlorpromazine, and family psychotherapy, the behavioral disturbances were further improved and the ear click was audible only on the right side and had started to fluctuate, disappearing spontaneously for days, reappearing spontaneously in stressful situations. Over time, the patient had developed a greater ability to inhibit the palatal tremor voluntarily and to induce it on request 
or by thinking about it, sometimes to attract attention, thus demonstrating voluntary control.

\section{Discussion}

Palatal tremor is an abnormal movement of the soft palate and was previously known as palatal myoclonus but more recently has been renamed palatal tremor because this corresponds better with the electrophysiological characteristics of the rhythmic pattern. The heterogeneous nature of palatal tremor covers a wide spectrum of clinical pictures, including symptomatic essential and psychogenic forms.

Secondary palatal tremor can be a consequence of vascular, infectious, degenerative, traumatic, or neoplastic lesions of the brainstem or cerebellum. This form is often associated with neurological deficits and, once present, persists throughout life and does not disappear during sleep or even in coma. Most symptomatic cases occur in adulthood, while a few cases have been reported in childhood in the course of Krabbe's disease, ${ }^{6}$ a cerebellar tumor, ${ }^{7}$ and encephalitis. ${ }^{8}$ In secondary palatal tremor, abnormal hypertrophy of the inferior olives has been detected, but its precise role in causing palatal tremor is controversial and not yet clearly demonstrated.

Recently Shaikh et $\mathrm{a}^{9,10}$ have shown, through a mathematical model, that oculopalatal tremor oscillations originate in the hypertrophic inferior olive and are amplified by learning in the cerebellum.

In essential palatal tremor, neurological investigations and brain neuroimaging are normal, while an ear click is present, perceived as objective tinnitus, that does not occur in the symptomatic form. Deuschl et al ${ }^{11}$ demonstrated that, in secondary palatal tremor, the movement of the soft palate is caused by contraction of the levator veli palatini and in essential palatal tremor, by contraction of the tensor veli palatini which induces secondary closure of the Eustachian tubes, causing a clicking sound that is audible by the examiner. Essential palatal tremor is more frequently bilateral, occurs mainly in children, and usually disappears when the patient's mouth is open and during sleep, although in some cases it persists even during sleep. The pathophysiology of essential palatal tremor remains unclear; it is generally benign, persists for months or years, and in children it tends to fluctuate and disappear spontaneously. Essential palatal tremor has been observed in monozygotic male twins, in one of whom symptoms developed at the age of 14 years and in the other at 37 years, suggesting a genetic etiology of the disorder. ${ }^{12}$ FernandezAlvarez ${ }^{13}$ included essential palatal tremor in the class of transient movement disorders, a heterogeneous group of primary pediatric movement disorders with a limited duration of expression over time, none or mild disability, and a spontaneously fading disorder. The diagnosis is clinical in the absence of biological markers.

Voluntary control of palatal tremor is possible in some cases of essential palatal tremor. In fact, the abnormal movement can be voluntarily induced or inhibited, and can also be discontinued when the patient is concentrating on motor and cognitive tasks, performing movements, reading, thinking, or doing calculations. Some authors have suggested that voluntary control of essential palatal tremor could be due to a certain degree of cortical control that is otherwise absent in the symptomatic forms, while other authors have suggested that it could be due to a psychogenic origin of the disorder.

Few cases classified as psychogenic palatal tremor have been reported in the literature. This form is often associated with psychopathology, responds to placebo and improves with psychotherapy. Furthermore, the movements have a variable frequency, and unlike the other forms of palatal tremor, increase during attention and decrease when the patient is distracted. Nevertheless, psychiatric symptoms, in particular anxiety disorders, have also been reported in the literature for some cases of essential palatal tremor.

After a review of the literature in relation to different forms of voluntary control, Samuel et $\mathrm{al}^{14}$ suggested that rather, than being a uniform pathogenic entity, essential palatal tremor may be a heterogeneous disorder including different forms; in particular, a form of conversion disorder, a tic disorder, or a series of completely involuntary movements, as described in most patients with essential palatal tremor. Moreover, the same authors suggested a correlation between secondary palatal tremor and essential palatal tremor, in view of the fact that functional magnetic resonance imaging studies have shown an activation of the inferior olives during palatal movements in the essential form. ${ }^{15}$ These studies suggest an involvement of the same structures in essential palatal tremor and secondary palatal tremor. In addition, in some cases of essential palatal tremor, an association with minor ailments has been reported, including respiratory infections, otitis, tonsillitis, fever, minor head trauma, and headache.

The clinical characteristics of our patient seem to support this hypothesis of a continuum between the different forms of palatal tremor (Table 1). He has a palatal tremor with an ear click, audible in both ears, which is usually 
Table I Clinical features of various types of palatal tremor

\begin{tabular}{|c|c|c|c|c|}
\hline & SPT & EPT & PPT & Case \\
\hline Etiology & $\begin{array}{l}\text { Brainstem, cerebellar, } \\
\text { basal ganglia }\end{array}$ & $?$ & $\begin{array}{l}\text { Psychological or } \\
\text { psychiatric disorders }\end{array}$ & $?$ \\
\hline Gender & $>$ Male & Male/Female & Male/Female & Male \\
\hline Age at onset & $>$ Adult & $>$ Children & Adult & I I-year-old \\
\hline Neurological examination & Abnormal & Normal & Normal & Hypotonia of facial muscles \\
\hline Ear click & Absent & Present & Present & Present \\
\hline Neuroimaging & Abnormal & Normal & Normal & Normal \\
\hline Voluntary control & No & Rarely & Yes & Yes \\
\hline Relationship to sleep & Yes & No & Variable & Yes \\
\hline Course & Continuous & Disappear spontaneously & Variable? & Continuous \\
\hline Side & Monolateral & Bilateral & $?$ & Bilateral \\
\hline
\end{tabular}

Abbreviations: EPT, essential palatal tremor; secondary palatal tremor; PPT, psychogenic palatal tremor.

present in the essential form but is rare in the symptomatic form. The palatal tremor persisted during sleep, which is common in the symptomatic form but rare in the essential form. Furthermore, neuroimaging studies did not document lesions in the brainstem or cerebellum, which are typically present in the symptomatic form. In addition, in our patient, pre-perinatal risk factors were present, namely a family history of psychiatric disorders, motor developmental delay, mental retardation, hypotonia of the facial muscles, and behavioral disturbances. These manifestations suggest symptomatic involvement of the brain. However, our patient was able to voluntarily induce or stop the tremor. Even if voluntary modulation of palatal tremor has been reported in literature in some cases of essential palatal tremor, our patient has developed possible voluntary control, suggesting the acquisition of special motor skills rather than an inhibition of involuntary movements, and this seems suggestive of a psychogenic etiology. ${ }^{16}$ Limited data are available about psychogenic movement disorders in children, but clinical findings suggesting a psychogenic cause similar to that in adults, including inconsistent character of the movements, exacerbation with stress and while paying attention, and disappearance when distracted, as well as a response to placebo and improvement with psychotherapy. The underlying psychiatric diagnosis was a conversion disorder in most of the cases described in literature. ${ }^{17}$ In our patient, the disorder also shared some features of psychogenic movement disorders, such as a discontinuous ear click, association with psychopathological disorders, an increase during periods of attention and disappearance at moments of distraction, as well as voluntary control of the movement.

In conclusion, the clinical characteristics of our patient suggest that there may be a neuropsychopathological continuum between different forms of palatal tremor, but this hypothesis requires further investigation, especially functional neuroimaging and genetic studies.

\section{Disclosure}

The authors report no conflict of interest in this work.

\section{References}

1. Deuschl G, Mischke G, Schenck E, Schulte-Mönting J, Lücking CH. Symptomatic and essential rhythmic palatal myoclonus. Brain. 1990; 113(Pt 6):1645-1672.

2. Lapresle J. Rhythmic palatal myoclonus and the dentato-olivary pathway. J Neurol. 1979;220(4):223-230.

3. Campistol-Plana J, Majundar A, Fernandez- Alvarez E. Palatal tremor in childhood: Clinical and therapeutic considerations. Dev Med Child Neurol. 2006;48(12):982-984.

4. Richardson SP, Mari S, Matsuhashi M, Hallett M. Psychogenic palatal tremor. Mov Disord. 2006;21(2):274-276.

5. Vaughn CE, Leff JP. The influence of family and social factors on the course of psychiatric illness. Br J Psychiatry. 1976;129:125-137.

6. Yamanouchi H, Kasai H, Sakuragawa N, Kurokawa T. Palatal myoclonus in Krabbe disease. Brain Dev. 1991;13(5):355-358.

7. Deuschl G, Jost S, Schumacher M. Symptomatic palatal tremor is associated with signs of cerebellar dysfunction. $J$ Neurol. 1996;243(7):553-556.

8. Baram TZ, Parke JT, Mahoney DH. Palatal myoclonus in a child: Herald of acute encephalitis. Neurology. 1986;36(2):302-303.

9. Shaikh AG, Hong S, Liao K, et al. Oculopalatal tremor explained by a model of inferior olivary hypertrophy and cerebellar plasticity. Brain. 2010;133(3):923-940.

10. Hong S, Leigh RJ, Zee DS, Optican LM. Inferior olive hypertrophy and cerebellar learning are both needed to explain ocular oscillations in oculopalatal tremor. Prog Brain Re. 2008;171:219-226.

11. Deuschl G, Toro C, Hallett M. Symptomatic and essential palatal tremor. 2. Differences of palatal movements. Mov Disord. 1994;9(6):676-678.

12. Kutukcu Y, Imirzalioglu N, Odabasi Z, Gokcil Z, Vural O. Essential palatal myoclonus in monozygotic male twins. $J$ Neurol. 2003;250 (7):885-886.

13. Fernández-Alvarez E. Movement disorders in children: Recent advances in management. Indian J Pediatr. 2009;76(5):531-536.

14. Samuel M, Kleiner-Fisman G, Lang AE. Voluntary control and a wider clinical spectrum of essential palatal tremor. Mov Disord. 2004;19(6):717-719. 
15. Deuschl G, Toro C, Valls-Solé J, Zeffiro T, Zee DS, Hallett M. Symptomatic and essential palatal tremor. 1. Clinical, physiological and MRI analysis. Brain. 1994;117(Pt 4):775-788.

16. Klein C, Gehsking E, Vieregge D. Voluntary palatal tremor in two siblings. Mov Disord. 1998;13(3):545-548.
17. Schwingenschuh P, Pont-Sunyer C, Surtees R, Edwards MJ, Bhatia KP Psychogenic movement disorders in children: A report of 15 cases and a review of the literature. Mov Disord. 2008;23(13):1882-1888.

\section{Publish your work in this journal}

Neuropsychiatric Disease and Treatment is an international, peerreviewed journal of clinical therapeutics and pharmacology focusing on concise rapid reporting of clinical or pre-clinical studies on a range of neuropsychiatric and neurological disorders. This journal is indexed on PubMed Central, the 'PsycINFO' database and CAS, and is the official journal of The International Neuropsychiatric Association (INA). The manuscript management system is completely online and includes a very quick and fair peer-review system, which is all easy to use. Visit http://www.dovepress.com/testimonials.php to read real quotes from published authors.

Submit your manuscript here: http://www.dovepress.com/neuropsychiatric-disease-and-treatment-journal 